\title{
POPULATION STUDY ARTICLE Prenatal second-hand smoke exposure and newborn telomere length
}

\author{
Bingqing Liu', Lulu Song ${ }^{1}$, Lina Zhang ${ }^{1}$, Mingyang $\mathrm{Wu}^{1}$, Lulin Wang ${ }^{1}$, Zhongqiang $\mathrm{Cao}^{2}$, Chao Xiong ${ }^{2}$, Bin Zhang ${ }^{2}$, Yuanyuan $\mathrm{Li}^{3}$,
} Wei Xia ${ }^{3}$, Shunqing $\mathrm{Xu}^{3}$ and Youjie Wang ${ }^{1}$

BACKGROUND: Cigarette smoking is associated with shorter telomere lengths in adults, but evidence on the effect of prenatal tobacco exposure is limited. We aimed to investigate the association between prenatal second-hand smoke exposure and newborn telomere length.

METHODS: We recruited 762 mother-newborn pairs from Wuhan Children's Hospital (Wuhan Maternal and Child Healthcare Hospital) between November 2013 and March 2015. Information on second-hand smoke exposure was obtained via questionnaires. Relative telomere length was measured in DNA extracted from umbilical cord blood. We used linear regression to assess the associations between prenatal second-hand smoke exposure and newborn telomere length.

RESULTS: In the fully adjusted model, prenatal second-hand smoke exposure was associated with $9.7 \%$ shorter newborn telomere length (percent difference: $-9.7 \%$; $95 \%$ confidence interval $(\mathrm{Cl})$ : $-15.0,-4.0$ ). The estimate for boys was lower (percent difference: $-10.9 \%$; $95 \% \mathrm{Cl}:-18.6,-2.5)$ than that for girls (percent difference: $-8.5 \%$; $95 \% \mathrm{Cl}:-15.8,-0.5)$, but the interaction term between newborn sex and prenatal second-hand smoke was not significant $(P=0.751)$.

CONCLUSIONS: This study demonstrated that prenatal second-hand smoke exposure may be a preventable risk factor for accelerated biological aging in the intrauterine stage, and further suggested possible sex differences in the susceptibility to prenatal second-hand smoke.

Pediatric Research (2020) 87:1081-1085; https://doi.org/10.1038/s41390-019-0594-2

\section{INTRODUCTION}

Telomere is a nucleoprotein complex at the chromosome end, which maintains genomic DNA stability. ${ }^{1}$ Telomere progressively shortens with each cell division and eventually reaches a critical length that triggers cellular senescence. ${ }^{1}$ Because telomere attrition is a key mechanism of cellular senescence, telomere length (TL) has long been considered as a marker of biological aging. A substantial body of studies indicated that shortened $T L$ in adulthood is associated with shorter lifespan and age-related diseases. $^{2,3}$ TLs are highly variable across individuals. It was reported that this inter-individual variation was originated early in life, while the lifestyle or environment in adulthood exerts a minor impact. ${ }^{4}$ Therefore, understanding the determinants of initial TL (TL of newborns) might provide insights into possible mechanisms underlying relationships between prenatal exposures and later health outcomes.

Cigarette smoking is a major risk factor of age-related diseases, ${ }^{5}$ and is associated with intensified oxidative stress and inflammation. 6,7 Both oxidative stress and inflammation are related to telomere shortening, ${ }^{8,9}$ leading to the hypothesis that cigarette smoking accelerates telomere attrition. It has been well established that active smoking is associated with shorter TL in adults. $^{10}$ Compared with active smoking, second-hand smoke (SHS) exposure is a more common and avoidable public health threat. Findings about the relationship between SHS exposure and TL among adults are inconsistent. ${ }^{11-13}$ Fetuses are more vulnerable to environmental threats than adults. However, the association between prenatal SHS exposure and newborn TL was poorly understood.

In China, the active smoking rate for women of childbearing age is very low $(0.7-1.6 \%){ }^{14}$ while researches showed that there were 38.9 to $75.1 \%$ non-smoking women exposed to SHS during pregnancy. ${ }^{15}$ The high prevalence of SHS exposure during pregnancy indicates an urgent need for the illustration of adverse impact of SHS exposure on fetus. In this study, we investigated the association between prenatal SHS exposure and newborn TL, with the hypothesis that prenatal SHS exposure was associated with shorter newborn TL.

\section{MATERIALS AND METHODS}

Participants

We recruited 762 mother-newborn pairs from Wuhan Children's Hospital (Wuhan Maternal and Child Healthcare Hospital), a maternity hospital in Wuhan city, Hubei province, China, between November 2013 and March 2015. The following inclusion criteria were applied: (a) during the first trimester of pregnancy ( $<16$ weeks); (b) singleton pregnancy; (c) residents of Wuhan City

\footnotetext{
${ }^{1}$ Department of Maternal and Child Health, School of Public Health, Tongji Medical College, Huazhong University of Science and Technology, Hangkong Road 13, 430030 Wuhan, Hubei, China; ${ }^{2}$ Wuhan Children's Hospital (Wuhan Maternal and Child Healthcare Hospital), Tongji Medical College, Huazhong University of Science and Technology, Hangkong

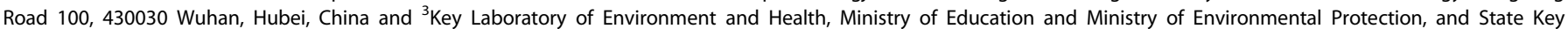
Laboratory of Environmental Health, School of Public Health (Incubation), Tongji Medical College, Huazhong University of Science and Technology, Wuhan, China Correspondence: Youjie Wang (wangyoujie@mails.tjmu.edu.cn)
} 
during pregnancy; (d) intention to have prenatal care and give birth at Wuhan Children's Hospital; (e) consent for participation in this study. After excluding 16 participants with DNA sample unavailable for TL measurement, there were 746 mother-newborn pairs included in the analysis.

The study protocol of this study was approved by the Medical Ethics Committee of Tongji Medical College and the Wuhan Children's Hospital. Signed informed consent was obtained from all participants.

\section{Data collection}

Maternal SHS exposure was collected via questionnaire, by asking "do you have SHS exposure during pregnancy?" SHS exposure was defined as non-smokers' exposure to the tobacco smoke for an average of at least $10 \mathrm{~min}$ per day. Active maternal smoking status was collected via questionnaires, by asking "do you have active cigarette smoking six months before pregnancy" and "do you have active cigarette smoking during pregnancy." Demographic information on age at delivery, education background (high school or below, college or above), and annual household income were collected via questionnaire. All the participants were interviewed face-to-face by trained nurses during the period of institutional delivery. Self-reported pre-pregnancy weight was collected at the first antenatal care visit (in the first trimester). Standing height was obtained by medical examination. Prepregnancy body mass index (BMI) was calculated as weight (kg) divided by height squared $\left(\mathrm{m}^{2}\right)$. The information on parity, gestational diabetes, and hypertensive disorders in pregnancy were obtained from medical records. Information on newborn sex, birth weight, and date of delivery were obtained from hospital delivery records. Low birth weight infant was defined as infant with birth weight $<2500 \mathrm{~g}$. Information on gestational age was estimated by women's last menstrual period, which was obtained from medical records. The preterm birth infant was defined as an infant with gestational age $<37$ weeks.

Umbilical cord blood collection and TL measurement At birth, umbilical cord blood samples were drawn in EDTA tubes. DNA was extracted from stored umbilical cord blood samples using the Wizard ${ }^{\oplus}$ Genomic DNA Purification (Promega Corporation, Madison, WI) following the manufacturer's instructions. Quantitative real-time polymerase chain reaction ( $q P C R$ ) was used for TL measurement. This method measured the relative TL by determining the T/S ratio of telomere repeat copy number $(T)$ and single-copy nuclear gene copy number (human $\beta$-globin) (S), and the $T / S$ ratio of the experimental sample was standardized with the reference pooled sample. The standard DNA pool consists of 50 DNA samples that were randomly selected from the 746 samples of this study. The four primers $5^{\prime}-3^{\prime}$ were as follows: telomere forward, 5'-ACACTAAGGTTGGGTTGGGTTGGGTT TGGGTTAGTGT-3'; telomere reverse, 5'-TGTTAGGTATCCCTATCCCT ATCCCTATCCCTATCCCTAACA-3'; reference gene forward, 5'-GT GCACCTGACTCCTGAGGAGA-3'; reference gene reverse, 5'-CCTT GATACCAACCTGCCCAG-3'. The reagents in the $10 \mu \mathrm{L}$ PCR were 1 $\mu \mathrm{L}$ of extracted DNA $(10 \mathrm{ng} / \mu \mathrm{L}), 5 \mu \mathrm{L}$ of KAPA SYBR ${ }^{\infty}$ FAST Master MIX 1× (KAPA Biosystems), and RNase free water. The final telomere primer concentrations were: telomere forward, $270 \mathrm{nM}$; telomere reverse, $900 \mathrm{nM}$. The final single copy gene primer concentrations were: HGB forward, 200 nM; HGB reverse, 200 nM. Samples were run in triplicate in a 384-well plate with the use of $\mathrm{Vii}^{\mathrm{TM}} 7$ Dx Real-Time PCR System (Applied Biosystems). The acceptable standard deviation (SD) for three $\mathrm{Ct}$ values was set at 0.3. If the SD of three $\mathrm{Ct}$ value was out of acceptable range, the measurement was repeated in a new 384-well plate. The thermal cycling profile were: $50^{\circ} \mathrm{C}$ for $2 \mathrm{~min}, 95^{\circ} \mathrm{C}$ for $3 \mathrm{~min}$, followed by 40 cycles of $95^{\circ} \mathrm{C}$ for $3 \mathrm{~s}$, and $60^{\circ} \mathrm{C}$ for $30 \mathrm{~s}$. A melting-curve analysis was performed at the end of each run to ensure the amplification specificity and absence of primer dimer. For the standard curve, the standard DNA pool was four-fold serial diluted to generate five solutions with concentrations ranging from 0.4 to $104 \mathrm{ng} / \mu \mathrm{L}\left(R^{2} \geq\right.$ 0.99). In order to assess GPCR amplification efficiency, we run a five-point serial dilution of standard reference genomic DNA sample on each run. The qPCR amplification efficiency was $108 \%$ for telomere and $103 \%$ for single-copy gene. We calculated coefficient of variation (CV) to assess the variability. The CV was calculated using the following formula: $(\sigma \div \mu) \times 100 \%$, where $\sigma$ and $\mu$ are standard deviation and mean value of the $C t$ values of single-copy gene of standard reference sample, respectively. The inter-run CV was calculated using $\mathrm{Ct}$ values of single-copy gene of standard reference sample in all qPCR assays. And the intra-run CV was calculated using $\mathrm{Ct}$ values of three replicates for single-copy gene of the standard reference genomic DNA sample in each 384well sample plate. The inter-run CV and mean intra-run CV were $4.1 \%$ and $3.0 \%$, respectively.

Statistical analysis

Categorical variables were expressed as proportions (\%), and continuous variables were expressed as means $\pm \mathrm{SD}$ )for normal distribution, or geometric mean (GM) and 95\% confidence interval (Cl) for skewed distribution. Differences in means and proportions between two categories of prenatal SHS exposure were tested by the analysis of variance or $x^{2}$ test. Linear regression models were employed to investigate the relationship between prenatal SHS exposure and In-transformed T/S ratio. Newborns without prenatal SHS exposure were treated as the reference group, and the percent difference in newborn TL was estimated as $e^{\beta}-1 \times 100 \%$. An unadjusted model was used to assess the association between SHS and newborn TL without adjustment for any covariate. We selected covariates based on a priori knowledge, including maternal age, pre-pregnancy BMI, education background, annual household income, parity, gestational diabetes, hypertensive disorders in pregnancy, infant sex, gestational age, birth weight, and date of delivery.

Because previous studies have suggested sex-specific relationship between prenatal environmental pollutants exposure and newborn $\mathrm{TL}^{16}{ }^{16}$ we inserted newborn sex $\times \mathrm{SHS}$ as an interaction term in the regression models to obtain $P$ value for interaction. The stratified analyses were employed to show the specific differences in percent changes of TL between boys and girls. To evaluate the robustness of our study, we performed sensitivity analyses excluding newborns with preterm birth and low birth weight, women $\geq 35$ years old, women with gestational diabetes and hypertensive disorders in pregnancy, and women with active cigarette smoking before or during pregnancy. All statistical analyses were performed using SAS 9.4 (SAS Institute Inc., Cary, NC). Statistical significance was set at $P<0.05$.

\section{RESULTS}

Characteristics of the participants according to prenatal SHS exposure are shown in Table 1. Of 746 eligible women, 244 $(32.7 \%)$ reported SHS exposure during pregnancy. Compared with women who had no exposure to SHS during pregnancy, women with SHS exposure were younger $(P=0.002)$ and had lower annual household income $(P=0.024)$. Compared with newborns without prenatal SHS exposure, those with SHS exposure had shorter umbilical cord blood TL $(P<0.001)$.

The distribution of fetal TL in two groups is shown in Fig. 1. The violin plot and box plot revealed that newborn TL was shorter in the non-SHS group, and the two groups had similar distribution of fetal TL.

We used two logistic regression models to assess the associations between prenatal SHS exposure and newborn TL (Table 2). In the unadjusted model, prenatal SHS exposure was associated with $10.5 \%$ shorter newborn TL (percent difference: $-10.5 \%$; $95 \% \mathrm{Cl}$ : $-15.7,-5.0)$. After adjustment for potential 
Table 1. Characteristics of the study participants according to prenatal second-hand smoke exposure

\begin{tabular}{|c|c|c|c|}
\hline \multirow[t]{3}{*}{ Characteristic } & \multicolumn{2}{|c|}{$\begin{array}{l}\text { Prenatal second-hand smoke } \\
\text { exposure }\end{array}$} & \multirow[t]{3}{*}{$P$ value } \\
\hline & No & Yes & \\
\hline & $N=502(67.3 \%)$ & $N=244(32.7 \%)$ & \\
\hline \multicolumn{4}{|l|}{ Maternal } \\
\hline Age (years) & $28.9 \pm 3.4$ & $28.1 \pm 3.1$ & 0.002 \\
\hline $\begin{array}{l}\text { Pre-pregnancy BMI } \\
\left(\mathrm{kg} / \mathrm{m}^{2}\right)\end{array}$ & $20.9 \pm 2.8$ & $21.0 \pm 2.9$ & 0.685 \\
\hline \multicolumn{4}{|l|}{ Education background } \\
\hline High school or below & $96(19.1 \%)$ & $61(25.0 \%)$ & 0.065 \\
\hline College or above & $406(80.9 \%)$ & $183(75.0 \%)$ & \\
\hline \multicolumn{4}{|l|}{$\begin{array}{l}\text { Annual household income } \\
\text { (Yuan) }^{\mathrm{a}}\end{array}$} \\
\hline$<50,000$ & $92(18.4 \%)$ & $52(21.4 \%)$ & 0.024 \\
\hline $50,000-100,000$ & $181(36.1 \%)$ & $106(43.6 \%)$ & \\
\hline$\geq 100,000$ & $228(45.5 \%)$ & 85 (35.0\%) & \\
\hline \multicolumn{4}{|l|}{ Number of parity } \\
\hline 1 & 427 (85.1\%) & 217 (88.9\%) & 0.148 \\
\hline$\geq 2$ & 75 (14.9\%) & 27 (11.1\%) & \\
\hline \multicolumn{4}{|l|}{$\begin{array}{l}\text { Smoking before } \\
\text { pregnancy }\end{array}$} \\
\hline No & 499 (99.4\%) & $243(99.6 \%)$ & 0.742 \\
\hline Yes & $3(0.6 \%)$ & $1(0.4 \%)$ & \\
\hline \multicolumn{4}{|l|}{$\begin{array}{l}\text { Smoking during } \\
\text { pregnancy }\end{array}$} \\
\hline No & $502(100 \%)$ & $244(100 \%)$ & \\
\hline Yes & $0(0 \%)$ & $0(0 \%)$ & \\
\hline \multicolumn{4}{|l|}{ GDM } \\
\hline No & $464(92.4 \%)$ & $229(93.9 \%)$ & 0.478 \\
\hline Yes & $38(7.6 \%)$ & $15(6.1 \%)$ & \\
\hline \multicolumn{4}{|l|}{ HDP } \\
\hline No & $489(97.4 \%)$ & $238(97.5 \%)$ & 0.915 \\
\hline Yes & $13(2.6 \%)$ & $6(2.5 \%)$ & \\
\hline \multicolumn{4}{|l|}{ Newborn } \\
\hline $\mathrm{TL}^{\mathrm{b}}, \mathrm{T} / \mathrm{S}$ ratio & $0.77(0.75,0.80)$ & $0.69(0.66,0.72)$ & $<0.001$ \\
\hline \multicolumn{4}{|l|}{ Sex } \\
\hline Male & $254(50.6 \%)$ & $129(52.9 \%)$ & 0.56 \\
\hline Female & $248(49.4 \%)$ & $115(47.1 \%)$ & \\
\hline \multicolumn{4}{|l|}{ PB } \\
\hline No & $488(97.2 \%)$ & 240 (98.4\%) & 0.337 \\
\hline Yes & $14(2.8 \%)$ & $4(1.6 \%)$ & \\
\hline \multicolumn{4}{|l|}{ LBW } \\
\hline No & $492(98.0 \%)$ & 239 (98.0\%) & 0.958 \\
\hline Yes & $10(2.0 \%)$ & $5(2.0 \%)$ & \\
\hline $\begin{array}{l}B M I \text { body mass index, } L B V \\
\text { gestational diabetes mellitu } \\
\text { aOne Yuan RMB is equal to } \\
{ }^{\mathrm{b}} \text { Geometric mean }(95 \% \mathrm{Cl})\end{array}$ & $\begin{array}{l}V \text { low birth weigh } \\
\text { s, HDP hypertensiv } \\
0.1477 \text { US\$ }\end{array}$ & $\begin{array}{l}\text { t, } P B \text { preterm bir } \\
\text { e disorders in preg }\end{array}$ & $\begin{array}{l}\text { th, GDM } \\
\text { gnancy }\end{array}$ \\
\hline
\end{tabular}

confounders, including maternal age, pre-pregnancy BMI, education status, annual household income, parity, gestational diabetes, hypertension disorders in pregnancy, infant sex, birth weight, gestational age, and date of delivery, this association was slightly attenuated, but still significant (percent difference: $-9.7 \%$; $95 \% \mathrm{Cl}$ : $-15.0,-4.0)$.

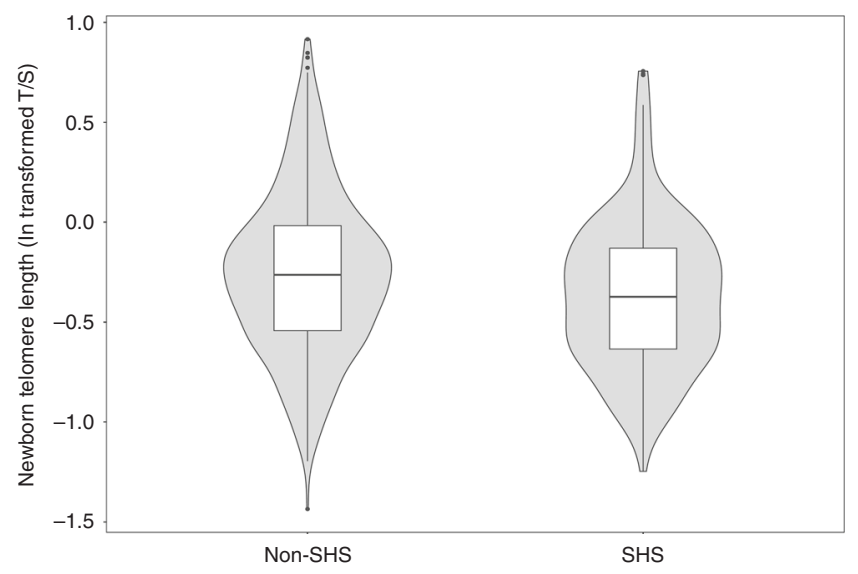

Fig. 1 The violin plot and box plot in distribution of newborn telomere length between two groups

We further did the stratified analysis by infant sex. After adjustment for potential confounders, the estimate for boys was lower (percent difference: $-10.9 \% ; 95 \% \mathrm{Cl}:-18.6,-2.5$ ) than that for girls (percent difference: $-8.5 \% ; 95 \% \mathrm{Cl}$ : $-15.8,-0.5$ ) (Table 2), but the interaction term between newborn sex and prenatal SHS was not significant $(P=0.751)$.

To evaluate the robustness of our study, we performed sensitivity analyses excluding newborns with preterm birth and low birth weight, women $\geq 35$ years, women with gestational diabetes and hypertensive disorders in pregnancy, and women with active cigarette smoking before or during pregnancy (Table 3). The associations between prenatal SHS exposure and umbilical cord blood TL did not materially changed.

\section{DISCUSSION}

In this study, we observed that compared with newborns without prenatal SHS exposure, those with SHS exposure had shorter umbilical cord blood TL. Besides, although the interaction term between newborn sex and prenatal SHS was not significant, the estimate for boys was lower than that for girls.

The association between active smoking and shorter TL has been well established in adults (Table 3 ). A meta-analysis included 30 studies have suggested an inverse relationship between packyears of smoking and TL. ${ }^{10}$ So far, there was only one study regarding the impact of prenatal tobacco exposure on cord blood TL. Salihu et al. ${ }^{17}$ conducted a study among 86 mother-newborn pairs, and they found a dose-response pattern that newborn TL for nonsmokers was longest, which is followed by that for passive smokers, and the shortest was that for active smokers. It was noteworthy that Salihu et al. ${ }^{17}$ did not control any potential confounders in their study. Besides, the small sample size, underprivileged study subjects with a high active smoking rate of Salihu's study might reduce the extension of these results to other population. There were two studies which investigated the association between prenatal tobacco exposure and early life TL among children population. Thall et al. ${ }^{18}$ conducted a crosssectional study among 104 children aged 4 to 14 years old, which suggested that children with prenatal tobacco exposure (maternal active and passive smoking during pregnancy) had shorter telomere than those without prenatal tobacco exposure. Consistent with Thall's study, a case-control study, which conducted among 196 Hong Kong Chinese children aged $<15$ years old, reported a negative dose-response relationship between the buccal epithelial cells $\mathrm{TL}$ and prenatal tobacco exposure duration. ${ }^{19}$ Because TL in later life is mainly determined by initial $\mathrm{TL}^{20}$ these two studies might support our findings. 
Table 2. Percent difference in newborn telomere length by prenatal second-hand smoke exposure

\begin{tabular}{|c|c|c|c|c|}
\hline Groups & \multicolumn{2}{|l|}{ Unadjusted } & \multicolumn{2}{|l|}{ Fully adjusted } \\
\hline \multicolumn{5}{|c|}{ Total population } \\
\hline Non-SHS & Referent & & Referent & \\
\hline SHS & $-10.5(-15.7,-5.0)$ & $<0.001$ & $-9.7(-15.0,-4.0)^{\mathrm{a}}$ & 0.001 \\
\hline \multicolumn{5}{|l|}{ Boys } \\
\hline \multicolumn{5}{|l|}{ Girls } \\
\hline Non-SHS & Referent & & Referent & \\
\hline SHS & $-9.0(-16.2,-1.0)$ & 0.028 & $-8.5(-15.8,-0.5)^{\mathrm{b}}$ & 0.038 \\
\hline
\end{tabular}

Table 3. Sensitivity analysis

\begin{tabular}{|c|c|c|c|c|c|}
\hline \multirow{2}{*}{$\begin{array}{l}\text { Sensitivity } \\
\text { analysis }\end{array}$} & \multirow[t]{2}{*}{$N$} & \multicolumn{2}{|l|}{ Unadjusted } & \multicolumn{2}{|l|}{ Fully adjusted } \\
\hline & & $\begin{array}{l}\text { Percent } \\
\text { difference } \\
(95 \% \mathrm{Cl})\end{array}$ & $P$ value & $\begin{array}{l}\text { Percent } \\
\text { difference } \\
(95 \% \mathrm{Cl})\end{array}$ & $P$ value \\
\hline \multicolumn{6}{|c|}{ Newborns without LBW and $\mathrm{PB}^{\mathrm{a}}$} \\
\hline Non-SHS & 488 & Referent & & Referent & \\
\hline SHS & 240 & $\begin{array}{l}-10.3 \\
(-15.9,-4.4)\end{array}$ & $<0.001$ & $\begin{array}{l}-9.8 \\
(-15.2,-4.0)\end{array}$ & 0.001 \\
\hline \multicolumn{6}{|c|}{ Women $<35$ years $^{\mathrm{a}}$} \\
\hline Non-SHS & 464 & Referent & & Referent & \\
\hline SHS & 234 & $\begin{array}{l}-10.6 \\
(-16.0,-4.9)\end{array}$ & $<0.001$ & $\begin{array}{l}-10.2 \\
(-15.7,-4.4)\end{array}$ & 0.001 \\
\hline \multicolumn{6}{|c|}{ Women without GDM and HDP } \\
\hline Non-SHS & 454 & Referent & & Referent & \\
\hline SHS & 224 & $\begin{array}{l}-10.9 \\
(-16.1,-5.3)\end{array}$ & 0.001 & $\begin{array}{l}-9.6 \\
(-15.3,-3.5)\end{array}$ & 0.002 \\
\hline \multicolumn{6}{|c|}{ Women without active smoking ${ }^{a}$} \\
\hline Non-SHS & 499 & Referent & & Referent & \\
\hline SHS & 243 & $\begin{array}{l}-10.4 \\
(-15.6,-4.8)\end{array}$ & $<0.001$ & $\begin{array}{l}-9.3 \\
(-14.7,-3.5)\end{array}$ & 0.002 \\
\hline
\end{tabular}

$C I$ confidence interval, SHS second-hand smoke, $L B W$ low birth weight, $P B$ preterm birth, GDM gestational diabetes mellitus, HDP hypertensive disorders in pregnancy

${ }^{a}$ Adjusted for maternal age, pre-pregnancy BMI, education status, annual household income, parity, GDM, HDP, infant sex, birth weight, gestational age, and date of delivery

${ }^{\mathrm{b}}$ Adjusted for maternal age, pre-pregnancy BMl, education status, annual household income, parity, infant sex, birth weight, gestational age, and date of delivery

Oxidative stress might be a potential mechanism underlying the associations between prenatal SHS exposure and newborn TL. Because of the high guanine content, telomeres are vulnerable to oxidative stress. Oxidative stress induce DNA breakage and accelerate the rate of telomere shorten with each cell division. ${ }^{21}$ SHS is a complex mixture containing various known carcinogens, teratogens, and toxins. ${ }^{22}$ Some of these chemical contaminants, such as nicotine, $\mathrm{CO}$, benzo(a)pyrene, lead, and cadmium, readily access to the fetus via placenta. ${ }^{23-27}$ The exposure to these toxic chemicals in cigarette smoke would induce oxidative stress, which accelerate telomere shortening in the fetus. In addition, endothelial dysfunction caused by cigarette smoke would intensify the oxidative stress, resulting in shortened telomeres. ${ }^{28}$

In this study, we also observed that the estimate for boys was lower than that for girls. However, it should be noted that the interaction between newborn sex and prenatal SHS was not significant, which indicated that this sex-specific association between prenatal SHS and newborn TL might be attributed to chance. Compared with female fetuses, male fetuses have lower level estrogen. ${ }^{29}$ Estrogen upregulates superoxide dismutase and glutathione peroxidase gene expression, resulting significantly higher antioxidant enzymes level in female fetus. ${ }^{30}$ Therefore, male fetuses might be more susceptible to oxidative stress caused by prenatal SHS exposure.

There were several limitations in our study. First, one major limitation is that prenatal SHS exposure was self-reported, which might introduce recall bias. However, previous studies had shown good reliability and validity of self-reported exposure to SHS among adults. ${ }^{31}$ Second, although extensive potential confounders were controlled in this study, we could not eliminate the unmeasured confounding caused by variables, such as maternal stress, nutritional status during pregnancy, and paternal age. These factors were reported to be related to offspring $\mathrm{TL}_{1}{ }^{32-34}$ and might confound the relationship between SHS and TL. Further studies with these informations are needed to confirm the finding of this study.

In this study, we found out prenatal SHS exposure was related with shorter newborn TL. Because the effect of early-life TL persists over the life course, the prenatal SHS exposure might have a lasting harmful effect on offspring's health outcomes in childhood and adulthood. ${ }^{20}$ China is the world's largest producer and consumer of tobacco. According Global Adult Tobacco Survey estimation in 2010, 52.9\% Chinese men were current smokers, whereas only $2.4 \%$ women were current smokers. ${ }^{14}$ The higher level of household smoking, poor ventilation, and overcrowding lead to widespread SHS exposure among Chinese women. ${ }^{14}$ The findings of our study adds to the growing body of evidence that prenatal exposure to SHS lead to adverse health impact on fetus. Effective intervention programs, which prevent SHS exposure during pregnancy, might improve longevity and life quality of offspring. 


\section{ACKNOWLEDGEMENTS}

This work was supported by the Hubei Province Health and Family Planning Scientific Research Project (grant number WJ2017Z001) and the National Natural Science Foundation of China (grant numbers 81273083, 91643207).

\section{AUTHOR CONTRIBUTIONS}

B.L. contributed to conception and design of the study, analysis and interpretation of data, and writing and revising the manuscript. L.S. contributed to collecting and analyzing data and revising the manuscript. L.Z., L.W., M.W., Z.C., B.Z., C.X., Y.L., W.X. and S.X. contributed to data collection and manuscript revising. Y.W. contributed to design of the study and revising the manuscript. All authors approved this manuscript to be published and agreed to be accountable for all aspects of this work.

\section{ADDITIONAL INFORMATION}

Competing interests: The authors declare no competing interests.

Publisher's note Springer Nature remains neutral with regard to jurisdictional claims in published maps and institutional affiliations.

\section{REFERENCES}

1. Blackburn, E. H., Epel, E. S. \& Lin, J. Human telomere biology: a contributory and interactive factor in aging, disease risks, and protection. Science 350, 1193-1198 (2015).

2. Bojesen, S. E. Telomeres and human health. J. Intern. Med. 274, 399-413 (2013).

3. Xi, H., Li, C., Ren, F., Zhang, H. \& Zhang, L. Telomere, aging and age-related diseases. Aging Clin. Exp. Res. 25, 139-146 (2013).

4. Benetos, A. et al. Tracking and fixed ranking of leukocyte telomere length across the adult life course. Aging Cell 12, 615-621 (2013).

5. Glantz, S. \& Gonzalez, M. Effective tobacco control is key to rapid progress in reduction of non-communicable diseases. Lancet 379, 1269-1271 (2012).

6. Burke, A. \& Fitzgerald, G. A. Oxidative stress and smoking-induced vascular injury. Prog. Cardiovasc Dis. 46, 79-90 (2003).

7. Goncalves, R. B. et al. Impact of smoking on inflammation: overview of molecular mechanisms. Inflamm. Res. 60, 409-424 (2011).

8. von Zglinicki, T. Oxidative stress shortens telomeres. Trends Biochem. Sci. 27, 339-344 (2002).

9. Jurk, D. et al. Chronic inflammation induces telomere dysfunction and accelerates ageing in mice. Nat. Commun. 2, 4172 (2014).

10. Astuti, Y., Wardhana, A., Watkins, J., Wulaningsih, W. \& Network, P. R. Cigarette smoking and telomere length: a systematic review of 84 studies and metaanalysis. Environ. Res. 158, 480-489 (2017).

11. Lu, L. et al. Association between exposure to second-hand smoke and telomere length: cross-sectional study of 1303 non-smokers. Int J. Epidemiol. 46, 1978-1984 (2017).

12. Hoxha, M. et al. Association between leukocyte telomere shortening and exposure to traffic pollution: a cross-sectional study on traffic officers and indoor office workers. Environ. Health 8, 41 (2009).

13. Wulaningsih, W., Serrano, F. E., Utarini, A., Matsuguchi, T. \& Watkins, J. Smoking, second-hand smoke exposure and smoking cessation in relation to leukocyte telomere length and mortality. Oncotarget 7, 60419-60431 (2016).
14. Li, Q., Hsia, J. \& Yang, G. Prevalence of smoking in China in 2010. N. Engl. J. Med. 364, 2469-2470 (2011).

15. Zhang, L. et al. Exposure to secondhand tobacco smoke and interventions among pregnant women in China: a systematic review. Prev. Chronic Dis. 12, E35 (2015).

16. Liu, H. et al. Prenatal exposure to perfluoroalkyl and polyfluoroalkyl substances affects leukocyte telomere length in female newborns. Environ. Pollut. 235, 446-452 (2018).

17. Salihu, H. M. et al. Impact of intrauterine tobacco exposure on fetal telomere length. Am. J. Obstet. Gynecol. 212, 205 e1-205 e8 (2015).

18. Theall, K. P., McKasson, S., Mabile, E., Dunaway, L. F. \& Drury, S. S. Early hits and long-term consequences: tracking the lasting impact of prenatal smoke exposure on telomere length in children. Am. J. Public Health 103(Suppl. 1), S133-S135 (2013).

19. Ip, P. et al. Prenatal tobacco exposure shortens telomere length in children. Nicotine Tob. Res. 19, 111-118 (2017).

20. Entringer, S., de Punder, K., Buss, C. \& Wadhwa, P. D. The fetal programming of telomere biology hypothesis: an update. Philos. Trans. R. Soc. Lond. Ser. B 373, 20170151 (2018).

21. Kawanishi, S. \& Oikawa, S. Mechanism of telomere shortening by oxidative stress. Ann. NY Acad. Sci. 1019, 278-284 (2004).

22. US Department of Health and Human Services. The Health Consequences of Smoking - 50 Years of Progress: A Report of the Surgeon General (US Department of Health and Human Services, Atlanta, 2014).

23. Luck, W., Nau, H., Hansen, R. \& Steldinger, R. Extent of nicotine and cotinine transfer to the human fetus, placenta and amniotic fluid of smoking mothers. Dev. Pharm. Ther. 8, 384-395 (1985).

24. Longo, L. D. The biological effects of carbon monoxide on the pregnant woman fetus, and newborn infant. Am. J. Obstet. Gynecol. 129, 69-103 (1977).

25. Arnould, J. P., Verhoest, P., Bach, V., Libert, J. P. \& Belegaud, J. Detection of benzo [a]pyrene-DNA adducts in human placenta and umbilical cord blood. Hum. Exp. Toxicol. 16, 716-721 (1997).

26. Goyer, R. A. Transplacental transport of lead. Environ. Health Perspect. 89, 101-105 (1990).

27. Piasek, M., Blanusa, M., Kostial, K. \& Laskey, J. W. Placental cadmium and progesterone concentrations in cigarette smokers. Reprod. Toxicol. 15, 673-681 (2001).

28. Celermajer, D. S. et al. Passive smoking and impaired endothelium-dependent arterial dilatation in healthy young adults. N. Engl. J. Med. 334, 150-154 (1996).

29. Reyes, F. I., Boroditsky, R. S., Winter, J. S. \& Faiman, C. Studies on human sexual development. II. Fetal and maternal serum gonadotropin and sex steroid concentrations. J. Clin. Endocrinol. Metab. 38, 612-617 (1974).

30. Vina, J. et al. Females live longer than males: role of oxidative stress. Curr. Pharm. Des. 17, 3959-3965 (2011).

31. Prochaska, J. J., Grossman, W., Young-Wolff, K. C. \& Benowitz, N. L. Validity of selfreported adult secondhand smoke exposure. Tob. Control 24, 48-53 (2015).

32. Entringer, S. et al. Maternal folate concentration in early pregnancy and newborn telomere length. Ann. Nutr. Metab. 66, 202-208 (2015).

33. Entringer, S. et al. Maternal psychosocial stress during pregnancy is associated with newborn leukocyte telomere length. Am. J. Obstet. Gynecol. 208, 134 e1-134 e7 (2013).

34. Unryn, B. M., Cook, L. S. \& Riabowol, K. T. Paternal age is positively linked to telomere length of children. Aging Cell 4, 97-101 (2005). 